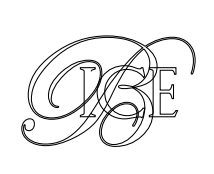

\title{
LA SOSTENIBILIDAD COMO HERRAMIENTA DE INTERNACIONALIZACIÓN: NUEVAS OPORTUNIDADES PARA LAS EMPRESAS
}

Las empresas españolas son cada vez más conscientes de su compromiso y responsabilidad con la sociedad y el entorno en el que operan, lo que se alinea perfectamente con la Agenda 2030, que les otorga un nuevo rol como actor de desarrollo. Ante este reto, ICEX ha desarrollado una nueva línea estratégica que toma la sostenibilidad como herramienta de internacionalización. En el presente artículo abordaremos cómo el binomio sostenibilidad e innovación actúa como vector de competitividad, generando un círculo virtuoso de «impacto positivo en el entorno-rentabilidad en el mercado internacional». Asimismo, analizaremos las nuevas líneas de trabajo, puestas en marcha por ICEX, con las que se apoya la internacionalización de la empresa desde una perspectiva más inclusiva y sostenible.

Palabras clave: Agenda 2030, Objetivos de Desarrollo Sostenible, negocios inclusivos, base de la pirámide, infraestructuras resilientes, cambio climático.

Clasificación JEL: F60, F61, F63, O10, Q50.

\section{Introducción}

Desde instancias y organismos públicos y privados dirigidos al apoyo a la internacionalización de la empresa, se traslada insistentemente a las pymes el papel clave que juega la internacionalización como elemento transformador de su negocio, generador de innovación y competitividad, fundamental para ampliar su mercado y contribuir al mismo tiempo a un

\footnotetext{
* ICEX España Exportaciones e Inversiones. Este artículo ha sido elaborado por María Peña Mateos, Directora de Cooperación Institucional y Coordinación.

Especial agradecimiento a la colaboración de Sara Ladra, Directora Adjunta de Cooperación y Sostenibilidad de ICEX hasta enero de 2018; David Muñoz, Jefe de Sector de dicho departamento; Rosa Angulo, Subdirectora de Internacionalización de la Empresa e Instituciones Multilaterales de ICEX; y Laura Gómez Clement, Jefe de Departamento en dicha Subdirección.

Versión de abril de 2018.
}

crecimiento más sostenible y equilibrado de la economía española.

En contadas ocasiones, sin embargo, se pone el énfasis en la capacidad de los procesos de internacionalización para generar valor en los países de destino. Y menos aún se incide en cómo dicha contribución positiva al desarrollo socioeconómico de un país puede revertir en una mejora de la posición competitiva de la empresa en los mercados internacionales, impulsar procesos de innovación en su interior, fortalecer su cadena global de valor, atraer y retener talento, atraer nuevas fuentes de financiación e inversión públicas y privadas y garantizar su aptitud para operar. Todo ello al tiempo que fideliza a sus clientes y proveedores, y accede a nuevos nichos de consumidores, que $\triangleright$ 
valoran que la estrategia de una empresa no solo se rija por su cuenta de resultados, sino también por el impacto social y ambiental que aporte su actividad allí donde opere. La generación de este círculo virtuoso — que va mucho más allá de la Responsabilidad Social Corporativa (RSC) tradicional- se traduce en la ventaja competitiva que ofrece integrar la sostenibilidad en el modelo de negocio y en la estrategia de internacionalización.

Cada vez hay más estudios que avalan la existencia de una relación de interdependencia entre sostenibilidad y rendimiento económico de las empresas. Tal como expone el informe de Deloitte 2030 Purpose: good business and a better future (2018), «...Raj Sisodia y otros investigadores demostraron en su obra Firms of Endearment que las empresas comprometidas con un "capitalismo consciente" generaron hasta catorce veces más ingresos que el resto de las empresas de S\&P 500 a lo largo de quince años. Desde entonces, los estudios académicos han demostrado que la ecoeficiencia y el compromiso con la sostenibilidad están relacionados con un excelente rendimiento económico». Si las empresas no son capaces de fusionar su interés particular con el interés general, su modelo de negocio estará amenazado a largo plazo (ECODES, 2018).

En este sentido, la Agenda 2030 de Naciones Unidas para el Desarrollo Sostenible ${ }^{1}$ realiza un enorme salto cualitativo en su concepción del desarrollo y los actores que inciden en él: por un lado, por su universalidad, comprometiéndonos a todos en sus diecisiete Objetivos de Desarrollo Sostenible ${ }^{2}$, con independencia del nivel de desarrollo del país de

\footnotetext{
https://www.un.org/sustainabledevelopment/es/la-agenda-de-desarrollosostenible/

2 http://www.undp.org/content/undp/es/home/sustainable-developmentgoals.html
}

referencia. Por otra parte, incorpora por primera vez al sector privado empresarial como un actor más de desarrollo, no solo como financiador, sino como un socio más, llamado a contribuir a dicha agenda y a la consecución de sus objetivos.

Desde septiembre de 2017, ICEX es miembro de la Red Española del Pacto Mundial de las Naciones Unidas y se ha adherido a los 10 Principios del Pacto Mundial ${ }^{3}$ en materia de derechos humanos, laboral y respeto al medioambiente.

El Pacto Mundial de las Naciones Unidas es la iniciativa mundial de referencia que trabaja en materia de sostenibilidad con el sector privado empresarial. Su ámbito de actuación con las empresas se centra fundamentalmente en:

1. Generar negocios responsables que alineen sus estrategias y operaciones con los 10 Principios del Pacto Mundial en derechos humanos, normas laborales, medioambiente y anticorrupción.

2. Liderar actuaciones estratégicas que permitan impulsar compromisos globales, como los Objetivos de Desarrollo Sostenible, poniendo particular énfasis en la innovación y la colaboración.

La incorporación de la sostenibilidad como línea de trabajo en el Plan Estratégico de ICEX 2017-2018 refleja nuestro compromiso con la Agenda 2030 y, por ende, con el sector privado para impulsar programas y actividades dirigidos a la incorporación de la sostenibilidad en las estrategias de negocio de nuestras empresas, contribuyendo con ello tanto a reforzar su competitividad internacional como su rol en la consecución de los Objetivos de Desarrollo Sostenible.

3 https://www.unglobalcompact.org/ 


\section{La estrategia de ICEX: hacia una internacionalización más sostenible}

Los objetivos que se propone alcanzar ICEX al integrar la sostenibilidad como un vector de competitividad para la internacionalización son los siguientes.

\section{Fomentar la diversificación de las exportaciones españolas hacia nuevos mercados y mejorar su posicionamiento en los tradicionales}

Uno de los objetivos del Plan Estratégico 2017-2018 de internacionalización de ICEX es la diversificación geográfica de las exportaciones, aumentando la presencia de las empresas españolas en sectores clave en nuevos mercados. Son precisamente estos mercados no tradicionales para nuestras exportaciones donde los retos del desarrollo son mayores (mercados emergentes y en desarrollo). La integración de la sostenibilidad en los procesos de internacionalización de las empresas en estos contextos se convierte en un factor clave para el éxito.

Asimismo, la sostenibilidad también puede resultar determinante para mantener un buen posicionamiento en los mercados desarrollados. Los procesos disruptivos de transformación digital y acceso a la información (big data) en los que estamos inmersos, unido al enorme peso que van tomando las redes sociales en los modelos actuales de creación de opinión, obligan a las empresas a abrir el núcleo de su actividad empresarial a los productores, trabajadores y consumidores, que pasan a convertirse en verdaderos «auditores» de la cadena de valor global de las empresas. La inclusión de los grupos de interés de la empresa en el proceso estratégico de toma de decisiones supone un fortalecimiento de la cadena de valor y una garantía de sostenibilidad de la estrategia de negocio en un medio-largo plazo. El modelo es aplicable en los países desarrollados (donde los consumidores han dejado de ser «simples consumidores», para convertirse también en «prescriptores de marca»), así como en el desarrollo de negocios inclusivos en los mercados de bajos ingresos que abordaremos posteriormente ${ }^{4}$.

Muy vinculado con lo anterior, adoptar la sostenibilidad en el modelo de negocio empresarial nos permite aumentar la confianza y fidelizar a los consumidores - con especial énfasis en los mercados de renta media y alta- a través de un desarrollo de la marca que sepa plasmar el compromiso de la empresa con la sostenibilidad social y medioambiental, diferenciando la misma de otras que no compartan ese mismo compromiso. Así, hoy en día podemos afirmar que se está produciendo un cambio de tendencia en los patrones de consumo, y casi el 50 por 100 del valor de una marca reside en aspectos intangibles como pueden ser la reputación, la imagen, las emociones que transmite a los consumidores y, cada vez más, la sostenibilidad. Así lo demuestra el estudio Loyalty Monitor 2017 realizado por el Instituto $\mathrm{DYM}^{5}$, cuyos resultados nos advierten de nuevas tendencias en cuanto a hábitos de consumo: junto a los clásicos factores como la marca (71 por 100) o la relación calidad-precio $\square$

\footnotetext{
4 Numerosas empresas energéticas o agroindustriales colaboran con las comunidades y administraciones locales en las regiones donde operan (i.e., comunidades indígenas, asociaciones de campesinos, mancomunidades, etcétera) antes de ejecutar sus proyectos. De esta forma, las empresas, además de obtener información de gran interés que les permita tener un mejor conocimiento sobre la zona, conocen también de primera mano las necesidades locales, pudiendo así adaptar sus proyectos a las necesidades de desarrollo locales. Ejemplo: Supracafe (http://www. supracafe.com/), compañía productora de café de alto valor añadido y modelo de producción comprometido con los productores locales como parte de su cadena de valor.

5 http://galanta.es/wp-content/uploads/2017/10/Loyalty-Monitor2017.-Clientes-fieles-e-infieles.compressed.pdf
} 
(47 por 100), los consumidores españoles empiezan a elegir un producto $u$ otro en función de factores como la sostenibilidad (12,5 por 100) o la identificación con los valores de esa marca (6,4 por 100).

\section{Favorecer el aprovechamiento de nuevos nichos de mercado por parte de las empresas españolas}

La empresa española ha demostrado con creces su competitividad internacional en sectores tan importantes para los Objetivos de Desarrollo Sostenible como la industria agroalimentaria (Objetivo 2); las infraestructuras del sector del agua (Objetivo 6, vinculado con su disponibilidad y gestión sostenible); las energías renovables (Objetivo 7, garantizar el acceso a una energía asequible, segura, sostenible y moderna para todos); y la lucha contra el cambio climático (Objetivo 13); el desarrollo de las infraestructuras (Objetivo 9, construcción de infraestructuras resilientes entre otros aspectos) o la gestión y desarrollo urbano (Objetivo 11, lograr que las ciudades sean seguras, eficientes y sostenibles).

Se trata ahora de dar un paso más: incorporar en el diseño, ejecución y desarrollo de los proyectos y modelos de negocio el impacto que estos generan en el entorno donde la empresa opera como variable estratégica de toma de decisiones. También en qué medida estimulan el desarrollo sostenible e inclusivo del país de referencia, contribuyendo así a los Objetivos 1 (poner fin a la pobreza en el mundo) y 10 (reducir la desigualdad en los países y entre ellos).

La integración de la sostenibilidad en la estrategia de negocio en sus tres vertientes (económica, social y medioambiental) viene justificada tanto como una forma de mitigación de riesgos como una vía para aprovechar importantes oportunidades de negocio, permitiendo la aparición de nuevos nichos de mercado derivados del compromiso de la empresa con su entorno.

Sectores como el agroalimentario, donde todos los elementos vinculados al proceso productivo (desde los sistemas de riego hasta los sistemas de procesamiento) pueden tomar en cuenta sus efectos en la seguridad alimentaria $^{6}$ o el cambio climático; o las telecomunicaciones, capaces de llegar a lugares más remotos y desarrollar servicios de telesalud o educación on-line, cobran una nueva dimensión bajo este enfoque ${ }^{7}$. En los sectores del transporte y la movilidad urbana, un importante foco de innovación está siendo el desarrollo de nuevos modelos de negocio que den respuesta a los problemas de movilidad y contaminación que enfrentamos hoy, cada vez más urgentes y complejos, muy especialmente en los países emergentes ${ }^{8}$.

Según la investigación llevada a cabo por su Comisión de Comercio y Desarrollo Sostenible, el Foro Económico de Davos estima que hasta 2030, los ODS generarán los sesenta mayores centros de actividad en el mercado, que equivaldrán a 12 billones de USD por año en ahoros comerciales y ganancias, tan solo en los cuatro sistemas económicos examinados: alimentos y agricultura, ciudades, energía y materiales, y salud y bienestar. Estos sectores, $\square$

6 GB Foods (https://www.thegbfoods.com/es) lanzó en 2008 al mercado cubitos de caldo enriquecidos con vitamina, deficitaria en la población local en África y Asia, lo que le permitió diferenciarse de sus competidores (Nestlé, Unilever, marcas locales...).

Ver, por ejemplo, la empresa SATEC (http://www.satec.es/), pionera en la prestación de asistencia sanitaria en zonas remotas en Perú y Colombia a través de soluciones de telesalud.

8 Empresas como Car2go (https://www.car2go.com/ES/es/) y Emov (https://emov.es/) ofrecen un servicio de coches de alquiler de pequeño tamaño alimentados por fuentes de energías renovables, para un periodo de tiempo corto, colocándose así a la vanguardia de la innovación sostenible en el sector de la movilidad. 
además, representan el 60 por 100 de la economía real, siendo asimismo ámbitos en los que las empresas españolas se sitúan en puestos de liderazgo mundial.

\section{Mejorar las oportunidades de acceso a la financiación}

Cada vez es más frecuente encontrar cláusulas sociales y medioambientales de obligado cumplimiento en los procesos de licitación internacional $y / o$ de instituciones financieras internacionales. Analizar bien estos requisitos y la normativa local e internacional que acompaña, así como cuantificar el impacto social y medioambiental derivado de la intervención, puede generar un importante valor añadido frente a los competidores ${ }^{9}$.

Más allá de la financiación multilateral, la integración de la sostenibilidad permite abrir las puertas a nuevas fuentes de financiación para las empresas, tanto públicas como privadas, fuentes procedentes de organismos multilaterales, instituciones financieras de desarrollo y agencias de desarrollo bilaterales, pero también puramente privadas como los fondos de inversión socialmente responsable (ISR) u otros inversores privados comprometidos en invertir su liquidez en productos que, además de generar una rentabilidad financiera, tengan un impacto social y medioambiental positivo en la sociedad.

\footnotetext{
9 Aunque existen muchos ejemplos, puede consultarse, p.e., el MAS (Marco Ambiental y Social del grupo Mundial, http://pubdocs.worldbank. org/en/345101522946582343/Environmental-Social-Framework-Spanish. pdf), que está formado por la visión de desarrollo sostenible del Banco Mundial, sus políticas y diez Normas Ambientales y Sociales (NAS). Aquí se estipulan los requisitos obligatorios para el Banco Mundial en relación con los proyectos que apoya a través del financiamiento para proyectos de inversión (IPF) y para los prestatarios.
}

\section{Fomentar procesos de innovación en sostenibilidad que mejoren la ventaja competitiva de las empresas}

La integración de la sostenibilidad permite, asimismo, activar procesos de innovación en el interior de la empresa, buscando adaptar las características y funcionalidades del producto ofertado o su forma de comercialización a nuevos contextos, sea en países desarrollados, emergentes o en desarrollo, posicionando dicha capacidad innovadora en la principal ventaja competitiva de la empresa. Los procesos de innovación derivados de la sostenibilidad implantados dentro de cada empresa pueden ser muy diferentes: habrá empresas que decidan innovar a nivel interno -en sus procesos productivos - para mejorar el impacto de su actividad en la sociedad y en el medio ambiente, pero sin que esto afecte a su modelo de negocio (i.e., incorporación del sello LEED ${ }^{10}$ en los edificios de referencia, como es el caso de la sede de ICEX en Castellana 278; introducción del teletrabajo, uso de energías renovables, cambios en la forma/tamaño del producto para reducir los costes de transporte, etcétera). Otras empresas pueden ir más allá y apostar por innovar tecnológicamente en la creación de productos o servicios sostenibles y novedosos que les permitan obtener un beneficio económico, a la vez que contribuyen a mejorar el medioambiente o la situación social de un sector de la población ${ }^{11}$. Por último, habrá $\triangleright$

10 El sello LEED (Leadership in Energy \& Enviromental Design) es un sistema de certificación de edificios sostenibles desarrollado por US Green Building Council. Se compone de un conjunto de normas sobre la utilización de estrategias encaminadas a todo tipo de edificios que se basan en la incorporación en el proyecto de aspectos relacionados con la eficiencia energética, el desarrollo sostenible de los espacios libres de la parcela y la selección de materiales y/o el uso eficiente del agua.

11 Otro ejemplo a unir a los anteriores es la empresa EcoAlf (https:// ecoalf.com/es/), que, con sus productos de moda sostenible, ha sabido cubrir un nicho de mercado de alta rentabilidad que demandaba un diseño ecológico de alta gama. 
empresas que perciban su actividad como parte de su entorno e innoven para mejorar su interacción con el mismo como base de su estrategia empresarial, colaborando con socios estratégicos no siempre habituales para el mundo empresarial (sociedad civil, organizaciones de productores, ONGD, universidades, etcétera) $)^{12}$.

\section{Líneas de acción para una internacionalización sostenible}

En todos los ámbitos, innovación y sostenibilidad aparecen como un binomio capaz de generar un círculo virtuoso de competitividad y rentabilidad en el mercado internacional. Bajo este enfoque, ICEX ha desarrollado distintas líneas de trabajo que tratan de responder al reto que afrontan las empresas en cada fase de su proceso de internacionalización:

1. Promocionar la integración de la sostenibilidad por parte de las empresas como eje de competitividad y herramienta de internacionalización, bien sea a través de foros o encuentros organizados por nuestros socios estratégicos, o a través de nuestros propios instrumentos de sensibilización y divulgación sobre la importancia de la internacionalización: ICEX Dinamiza, revista El Exportador (p. e., Negocios inclusivos. Elegir la vía social) ${ }^{13}$ o el programa de televisión, producido

\footnotetext{
12 Por ejemplo, la start-up española Frogtek (http://frogtek.org/), que ha desarrollado una aplicación denominada Tienda Teck dirigida a pequeños comercios en América Latina, que, además de poder usarse como terminal de venta, facilita la mejora de sus stocks y ventas y permite obtener métricas empresa social con ánimo de lucro centrada en el desarrollo de aplicaciones de productividad en dispositivos móviles para pequeños comerciantes de países en desarrollo.

13 https://www.icex.es/icex/es/Navegacion-zona-contacto/revista-elexportador/observatorio2/REP2017761148.html
}

por ICEX y emitido por RTVE, Conecta con el Mercado, que incluye casos de éxito de negocios inclusivos en varios capítulos (Colombia, Filipinas y México) ${ }^{14}$.

2. Reforzar dicha sensibilización a través de instrumentos que permitan a la empresa acercarse a la Agenda 2030 desde el punto de vista práctico, mediante herramientas de autodiagnóstico ${ }^{15}$ o una guía práctica sobre «Cómo atender nuevos mercados a través de modelos de negocio inclusivos y sostenibles ${ }^{16}$.

3. Potenciar los sectores susceptibles de abordar la sostenibilidad como factor diferencial a través de los servicios de información y de inteligencia económica. En 2017 se publicaron 17 estudios de mercado vinculados con energías renovables, 14 con la gestión de residuos o 16 sobre la gestión y tratamiento de aguas, por poner solo algunos ejemplos de sectores clave en la internacionalización sostenible. Con ello tratamos de abordar nuevos mercados y nuevos sectores, respondiendo además a una demanda de servicios cada vez más personalizada por parte de nuestros clientes. En el medio plazo esperamos poder también incorporar una primera aproximación de análisis de impacto en los estudios de mercados sectoriales elaborados por nuestra Red de Oficinas Económicas y Comerciales. La importancia de trabajar en estos ámbitos viene determinada $\triangleright$

\footnotetext{
14 http://www.rtve.es/alacarta/videos/conecta-con-el-mercado/conectamercado-colombia/4489098

15 https://www.icex.es/icex/es/navegacion-principal/todos-nuestrosservicios/programas-y-servicios-de-apoyo/icex-impact/que-ofrecemos/ autodiagnostico/index.html?utm_source=banner-interno\&utm_medium = icex-es\&utm_campaign=autodiagnostico\&utm_term=boton-impact\&utm_ content $=226-56$

16 https://www.icex.es/icex/wcm/idc/groups/public/documents/ documento_anexo/mde3/nzm1/ edisp/dax2017735778.pdf
} 
también por la demanda de nuestras empresas: Asia, Oriente Medio y Oceanía son el primer destino de demanda de nuestros servicios personalizados $(30,7$ por 100), seguido por África (23,6 por $100)$, ambas áreas con gran potencial en este ámbito.

4. Apoyar proyectos innovadores ante retos globales y contribuir a dar visibilidad a los esfuerzos de las empresas españolas en el ámbito de la sostenibilidad para que Made in Spain sea identificado como una marca-país sostenible. Entre las actividades de 2017 pueden mencionarse algunas como la jornada Integra Municipios (Antioquía, Colombia); las jornadas Ecosystem Engagement o Climate Technology Center and Network (Madrid); la jornada sobre tratamiento de residuos (Atenas, Grecia) o la jornada sobre Smart Cities en la Expo World Congress el pasado mes de noviembre en Barcelona.

5. Capacitar y acompañar a la empresa en el desarrollo de negocios inclusivos. Los mercados de base de la pirámide o de bajos ingresos constituyen una clara oportunidad de negocio para la empresa española. El programa IMPACT $+{ }^{17}$, que abordaremos posteriormente en el apartado 3 de este artículo, ha sido el primero creado por ICEX dirigido específicamente al tratamiento diferencial de estos mercados.

6. Fortalecer la capacidad de las empresas para cumplir con los requisitos de sostenibilidad que requieren las instituciones multilaterales, a través de actividades de capacitación en materia de:

17 https://www.icex.es/icex/es/navegacion-principal/todos-nuestrosservicios/programas-y-servicios-de-apoyo/icex-impact/objetivos/index. html
- Política de gestión de riesgos de las instituciones financieras y la sostenibilidad.

- Elaboración de mejores propuestas técnicas, adaptadas a los requisitos de cada uno de los organismos financiadores.

Entre las actividades desarrolladas en 2017 destacan algunas como la jornada Infraestructura y sostenibilidad: el reto de las multilaterales, celebrada en junio con el Banco Mundial; o el seminario Addressing Reputational Risks and Improving Compliance in Multilateral Projects, celebrado el 9 de junio en Madrid, donde se puso especial énfasis en la importancia del cumplimiento de los códigos éticos en el entorno multilateral o los llamados partenariados con instituciones financieras internacionales celebrados en Bogotá, El Cairo, Yangón y Manila, y a los que seguirán Buenos Aires, Túnez y Uzbekistán en 2018.

7. Establecer alianzas estratégicas con socios relevantes en el ámbito de la sostenibilidad. Así, para conseguir el éxito de esta nueva política estratégica es necesario que ICEX actúe de forma coordinada con socios estratégicos como son AECID, la Red Española del Pacto Mundial, CDTI o COFIDES, universidades y ecosistemas de innovación social (Impact Hub; Centro de Innovación y Tecnología para el Desarrollo Humano de la Universidad Politécnica, etcétera), participando en grupos de trabajo y foros, y creando alianzas que permitan maximizar sinergias en proyectos desarrollados conjuntamente. Complementariamente, ICEX se propone introducir la sostenibilidad en su relación con las $\square$ 
asociaciones sectoriales en aquellos sectores más relevantes para la Agenda 2030.

\section{Los mercados de bajos ingresos como oportunidad de negocio real: el programa IMPACT+}

Los modelos tradicionales de apoyo a la internacionalización de la empresa se han centrado en los segmentos socioeconómicos medios y altos, con capacidad adquisitiva para absorber productos y servicios de alto valor añadido y elevado precio. No obstante, esta población apenas supone el 30 por 100 de la población mundial. Por otro lado, según estimaciones realizadas por la Corporación Financiera Internacional (IFC, por sus siglas en inglés) del Grupo del Banco Mundial ${ }^{18}$, existen alrededor de 4.000 millones de personas con una capacidad de gasto inferior al equivalente a 8 USD/día (unos 3.000 USD al año en paridad de poder adquisitivo), la llamada Base de la Pirámide (BoP). Este enorme segmento de la población mundial vive en países en desarrollo de Asia, África Subsahariana, Europa Oriental y América Latina y el Caribe, con un crecimiento económico por encima de la media mundial y con una demografía joven con elevados ratios de natalidad y de urbanización, ambos indicadores del crecimiento potencial de la demanda agregada. Aunque tradicionalmente desatendida como potencial consumidor, la BoP en su conjunto representa un poder de compra potencial de 5 billones de USD aproximadamente. Las

18 «Los siguientes 4 mil millones. Tamaño del mercado y estrategia de negocios en la Base de la Pirámide», IFC. www.wri.org/thenext4billion proyecciones demográficas apuntan a que en 2050 el 87 por 100 de la población mundial estará concentrada en África, Asia y Latino América ${ }^{19}$.

De acuerdo al ya mencionado estudio de la Corporación Financiera Internacional, el mercado de mayor tamaño (medido por el gasto de los hogares) es sin duda el agroalimentario (2,36 billones de USD), seguido por el de la vivienda (508.000 millones de USD), energía (434.000 millones de USD), transporte (298.000 millones de USD), salud (243.000 millones de USD) y TIC (206.000 millones de USD).

Ahora bien, abordar este mercado, con independencia de su potencial, no es tarea fácil. La llamada BoP cuenta con algunas particularidades que nos obligan a innovar en la forma en que esta es abordada ${ }^{20}$ :

- Necesidades básicas no cubiertas, porque no pueden acceder a los sistemas de comercialización y pago tradicionales (distribución moderna versus tradicional; bajo grado de bancarización, limitado acceso a la electricidad y agua potable; etcétera).

- Dependencia de trabajos informales y de subsistencia, unidos a otras fuentes de ingresos irregulares, como son las remesas de emigrantes. Ello hace que su capacidad de compra y, por tanto, su ciclo de consumo sean enormemente fluctuantes.

- Aparición de una prima de la pobreza por bienes o servicios básicos, en gran medida por el ineficiente funcionamiento $D$

19 World Population Prospects (ONU). Revisión 2015.

20 Estudio sobre la internacionalización en mercados de bajos ingresos, realizado para ICEX por la consultora Roots for Sustainability en 2016. 
de los mercados (altos derechos de aduana, mercados oligopolizados, con pocos oferentes pero múltiples intermediarios en cada fase de la cadena de valor; ausencia de infraestructuras que incrementan exponencialmente los costes de transporte, etcétera).

- Por último, nos enfrentamos a modelos socioeconómicos cuyas claves no son evidentes bajo estándares tradicionales: p.e., jerarquías y grupos de poder en modelos sociales tradicionales; conocimiento de los elementos reputacionales que pueden incidir en los hábitos de consumo; sistemas comunales o de propiedad colectiva cuya comprensión es imprescindible para dotar de seguridad jurídica en muchos sectores a la inversión (agrario y minería, entre otros), etcétera.

La lista de factores diferenciadores podría ser mucho más larga, pero los anteriormente mencionados son suficientes para justificar la necesidad de convertir a nuestros consumidores, proveedores o distribuidores (provenientes de la misma BoP) en aliados estratégicos en el proceso de diseño y desarrollo del modelo de negocio. La cocreación así entendida —como primera condición necesaria para tener éxito en estos mercados- es la forma más eficiente de garantizar un conocimiento profundo de la realidad que queremos abordar, su rentabilidad y su sostenibilidad en el tiempo. La segunda condición necesaria es la innovación en productos, procesos, medios de pago y canales de distribución para convertir oportunidades de negocio potenciales en oportunidades de negocio reales, propiciando además procesos locales de innovación y actuando como mecanismo de inclusión de la población beneficiada que, de otra manera, no podría cubrir esas necesidades ${ }^{21}$.

Cada vez un mayor número de empresas españolas, conscientes del potencial que ofrecen los mercados BoP, encuentran los incentivos económicos necesarios dentro de sus estrategias de expansión para realizar proyectos pilotos, lanzar nuevos negocios o extender líneas de producto de sus negocios actuales para servir a estos mercados. No obstante, todavía hay mucho camino por recorrer, en parte porque existe un importante déficit de conocimiento por parte de las empresas españolas tanto sobre el potencial como sobre cómo adaptar mejor los modelos de negocio tradicionales a las particularidades de estos mercados $^{22}$.

ICEX, como ventana a la internacionalización de las empresas españolas, puede y quiere contribuir a apoyarlas en este desafío. En este sentido, resulta crucial realizar una caracterización de estos mercados y de los sectores de oportunidad que ofrecen a las empresas españolas, así como identificar, en base a sus necesidades y demandas, las mejores herramientas e instrumentos para articular este apoyo de manera efectiva.

\footnotetext{
21 Algunos ejemplos pueden ser ilustrativos: p.e., un modelo de electrificación rural será solo sostenible económicamente si, simultáneamente, al dotar de electricidad lugares rurales remotos, la empresa desarrolla un sistema de pago accesible, p.e., mediante un prepago por móvil. De la misma forma, el modelo de negocio de una empresa agroindustrial en un país donde no existen derechos de propiedad individual de la tierra será más sostenible social y económicamente cuanto mayores serán los niveles de inclusión de las comunidades locales en el proceso de producción y distribución. Otros ejemplos pueden ser: innovación en productos alimenticios que incorporen componentes nutricionales específicos o se adapten a las carencias existentes, como, p.e., acceso continuo a la electricidad o inexistencia de medios de almacenamiento en condiciones salubres, desarrollo de sistemas de pago a través del móvil, nuevos sistemas de packaging, etcétera.

22 La Guía práctica sobre estrategias para atender nuevos mercados a través de modelos de negocio inclusivos y sostenibles (https://www. icex.es/icex/wcm/idc/groups/public/documents/documento_anexo/ mde3/nzm1/ edisp/dax2017735778.pdf), publicada por el ICEX, responde a gran parte de estas cuestiones, entre ellas las características de mercado y cómo abordarlo.
} 


\subsection{Programa ICEX IMPACT+}

El programa ICEX IMPACT $+{ }^{23}$ persigue precisamente este objetivo de apoyar a las empresas españolas para que, en su proceso de búsqueda de nuevos mercados o nuevos nichos de actividad, desarrollen soluciones innovadoras en materia de sostenibilidad social y/o medioambiental que den respuesta a los retos globales en materia de desarrollo sostenible. El programa ofrece los siguientes servicios:

1. Identificación de oportunidades concretas de negocios inclusivos y sostenibles en estos mercados.

2. Acompañamiento a las empresas en sus procesos de prospección de estas oportunidades de negocio, a través de actividades dirigidas a:

- Conocer de primera mano las oportunidades de negocio identificadas.

- Establecer contactos con organismos públicos, comunidades y organizaciones locales, así como otros socios no tradicionales para la empresa presentes en el país, vinculados a dichas oportunidades.

- Estructurar modelos de negocios, junto con los socios locales y los potenciales clientes, a través de talleres de innovación y cocreación, que hagan rentables estas oportunidades para la empresa y que resulten, además, negocios socialmente inclusivos y medioambientalmente sostenibles.

3. Servicios de asesoramiento personalizado durante el proceso posterior de puesta en marcha del negocio en el país, una vez identificadas las necesidades de las

23 https://www.icex.es/icex/es/navegacion-principal/todos-nuestrosservicios/programas-y-servicios-de-apoyo/icex-impact/objetivos/index.html empresas, con base en las visitas y los potenciales modelos de negocio que se han identificado en los talleres.

Así, en 2017 ICEX ha realizado con gran éxito una primera edición de este innovador programa en Colombia, con la participación de trece empresas españolas de seis sectores diferentes (tecnología agrícola, comercialización y transformación de bienes agrícolas, energías renovables, ingeniería/consultoría, potabilización de agua y turismo sostenible) y con el apoyo directo y fundamental de la Oficina Económica y Comercial de la Embajada de España en Bogotá.

En el marco de esta primera edición piloto del programa ICEX IMPACT+, y tras la realización de las fases de identificación de oportunidades concretas de negocios inclusivos y sostenibles en varias regiones de Colombia y de acompañamiento a las empresas participantes para la realización de una prospección de estas oportunidades, se ha conseguido poner en marcha seis modelos de negocio diferentes. Estos modelos de negocio se han estructurado de forma directa entre las empresas y el resto de los agentes intervinientes en la oportunidad (organismos públicos locales, agencias multilaterales, comunidades y organizaciones de productores, etcétera) a través de procesos de cocreación dirigidos por responsables del programa ICEX IMPACT+ y, en muchos de ellos, intervienen varias empresas españolas de sectores diferentes, aprovechándose así interesantes complementariedades y sinergias surgidas.

En 2018, además de hacerlo en Colombia, ICEX continuará apoyando a través del programa ICEX IMPACT+ a las empresas españolas en sus procesos de internacionalización mediante modelos de negocio sostenibles e inclusivos en nuevos mercados en Latinoamérica y en el norte de África. 


\section{Conclusiones}

ICEX España Exportaciones e Inversiones ha asumido el reto lanzado por Agenda 2030 de convertir los retos globales en oportunidades de negocio, con un triple fin: en primer lugar, aumentar la presencia de la empresa española en nuevos nichos y sectores; en segundo lugar, favorecer la generación de ecosistemas que apoyen la innovación para la internacionalización; y, por último, afianzar el papel del sector privado en la consecución de los Objetivos de Desarrollo Sostenible. Con ello esperamos contribuir a los trabajos del Grupo de Alto Nivel (GAN) creado por la Comisión Delegada del Gobierno para Asuntos Económicos y a la preparación del Examen Nacional Voluntario (ENV) de España, que tendrá lugar en julio de 2018 y que dará indiscutiblemente un importante impulso a la implementación de la Agenda 2030 en nuestro país.

\section{Bibliografía}

[1] AZEVEDO, M. et al. (2015). Un mercado creciente de 750 mil millones de USD. Washington DC: Banco Interamericano de Desarrollo (BID).

[2] BUSINESS y SUSTAINABLE DEVELOPMENT COMMISSION. DAVOS FORUM (2017). Mejores negocios: Un mundo mejor.

[3] DELOITTE (2017). 2030 Purpose: Good business and better future. La sostenibilidad en la estrategia de las empresas españolas.

[4] FUNDACIÓN COTEC PARA LA INNOVACIÓN (2017). «La innovación social en España: Iniciativas para el desarrollo y la equidad», en Informe COTEC 2017.

[5] HAMMOND, A.; KRAMER, W. y KATZ, R. (2007). The next 4 billion. Washington DC: World Resources Institute and International Finance Corporation.
[6] ICEX, ESPAÑA EXPORTACIÓN E INVERSIONES (2016). Estudio sobre la internacionalización en mercados de bajos ingresos.

[7] ICEX, ESPAÑA EXPORTACIÓN E INVERSIONES (2017). Plan Estratégico 2017-2018.

[8] ICEX, ESPAÑA EXPORTACIÓN E INVERSIONES (2017). Guía práctica sobre Estrategias para atender nuevos mercados a través de modelos de negocio inclusivos y sostenibles.

[9] INTERNATIONAL FINANCE CORPORATION y WORLD BANK GROUP (2005). Global consumption data for inclusive business.

[10] JENKINS, B. e ISHIKAWA, E. (2009). Business Linkages: Enabling access to markets at the Base of the Pyramid. International Finance Corporation, International Business Leaders Forum and the CSR Initiative at the Harvard Kennedy School.

[11] JENKINS, B. e ISHIKAWA, E. (2010). Scaling up Inclusive Business: Advancing the knowledge and action agenda.

[12] KARRAMCHANDANI, A.; KUBZANSKY, M. y LALWANI, N. (2011). The Globe: Is the Bottom of the Pyramid Really for you? Harvard Business Review.

[13] KRÖDER, H. y DE VRIES, J. (2015). Sustainable profit for SMEs.

[14] POLAK, P. y WARWICK, M. (2013). The business solution to poverty: Designing products and services for three billion new customers.

[15] PORTER, M. y KRAMER, M.R. (2011). Creating Shared Value. Harvard Business Review.

[16] PRAHALAD, C.K. y HART, S. (2002). The fortune at the Bottom of the Pyramid.

[17] RED ESPAÑOLA DEL PACTO MUNDIAL (2016). El sector privado ante los Objetivos de Desarrollo Sostenible.

[18] VEGLIO, F. (2016). Delivering on Sustainable Development Goals.

[19] VIÑUALES, V. (2018). «Diálogo sobre los retos y oportunidades del sector agroalimentario» en la jornada El sector agroalimentario ante los Objetivos de Desarrollo Sostenible. Madrid, 13 de abril de 2018. 